Journal of Applied ANALysis

Vol. 9, No. 1 (2003), pp. 123-129

\title{
FUNCTIONS OF TWO VARIABLES WHOSE VERTICAL SECTIONS ARE EQUIDERIVATIVES
}

\author{
K. CHMIELEWSKA \\ Received April 4, 2001 and, in revised form, April 8, 2002
}

\begin{abstract}
We examine functions of two variables whose all vertical sections are equiderivatives. In particular we show that a bounded function whose horizontal sections are strongly measurable and vertical sections are equiderivatives, is strongly measurable. The theorems we prove are generalizations of the results of Z. Grande [3].
\end{abstract}

Let $\mathbb{R}$ and $\mathbb{N}$ denote the real line and the set of positive integers, respectively. Let $(X, \mathcal{M})$ be a measurable space and let $\mathcal{I} \subset \mathcal{M}$ be a proper $\sigma$-ideal of subsets of $X$. Assume that $Z$ is a Banach space.

Let $h: X \rightarrow Z$. Recall that $h$ is measurable, if $h^{-1}(U) \in \mathcal{M}$ for every open set $U \subset Z$. In [2], I introduced the following two kinds of measurability of a function. We say that $h$ is nearly simple, if there exist elements $\alpha_{1}, \alpha_{2}, \ldots \in Z$ and a sequence of pairwise disjoint sets $A_{1}, A_{2}, \ldots \in \mathcal{M}$ such that $h=\alpha_{n}$ on $A_{n}$ for each $n$, and $X=\bigcup_{n=1}^{\infty} A_{n}$. We say that $h$ is strongly measurable with respect to $(\mathcal{M}, \mathcal{I})$, if there exists a sequence of nearly simple functions $\left(h_{n}\right)$ and a set $A \in \mathcal{I}$ such that $h_{n} \rightarrow h$ on $X \backslash A$.

2000 Mathematics Subject Classification. 26B05, 26B99, 26A15.

Key words and phrases. Derivative, equiderivatives, strong measurability.

Supported by Bydgoszcz Academy 2000.

ISSN 1425-6908 C) Heldermann Verlag. 
Remark 1. Clearly Bochner integrable functions are strongly measurable, because they are pointwise limits of sequences of simple functions. Moreover strongly measurable functions are measurable. Easy examples show that the converse implications do not hold.

Remark 2. Usually in functional analysis (see, e.g., [6]) one considers a different kind of strong measurability, namely one assumes that there exists a sequence of simple functions $\left(h_{n}\right)$ (i.e., measurable functions with finite range) and a set $A \in \mathcal{I}$ such that $h_{n} \rightarrow h$ on $X \backslash A$. If we can find a $\sigma$-finite measure on $(X, \mathcal{M})$, then these two notions coincide.

We will need the following property of strongly measurable functions.

Proposition 1. Let $h: X \rightarrow Z$ be strongly measurable with respect to $(\mathcal{M}, \mathcal{I})$. Then

for each $\varepsilon>0$ and each $A \in \mathcal{M} \backslash \mathcal{I}$ there is a set $B \in \mathcal{M} \backslash \mathcal{I}$ with $B \subset A$ such that $\operatorname{osc}_{B} h<\varepsilon$,

where $\operatorname{osc}_{B} h=\sup \{\|h(x)-h(y)\|: x, y \in B\}$.

Proof. Let $\varepsilon>0$ and $A \in \mathcal{M} \backslash \mathcal{I}$. By [2, Corollary 3], there is a set $S \in \mathcal{I}$ such that $h(X \backslash S)$ is a separable subspace of $Z$. So, there are $z_{1}, z_{2}, \ldots \in Z$ such that

$$
h(X \backslash S) \subset \bigcup_{n=1}^{\infty} K_{n},
$$

where $K_{n}=\left\{z \in Z:\left\|z-z_{n}\right\|<\varepsilon / 3\right\}$. Thus

$$
A=(A \cap S) \cup \bigcup_{n=1}^{\infty}\left(A \cap h^{-1}\left(K_{n}\right)\right) .
$$

Since $A \notin \mathcal{I}$, there is an $n \in \mathbb{N}$ such that $B=A \cap h^{-1}\left(K_{n}\right) \notin \mathcal{I}$. Clearly $B \in \mathcal{M}$ and $\operatorname{osc}_{B} h \leq 2 \varepsilon / 3<\varepsilon$.

Remark 3. Recall that if condition (ccc) is fulfilled in $(X, \mathcal{M}, \mathcal{I})$ (i.e., if each family of pairwise disjoint elements of $\mathcal{M} \backslash \mathcal{I}$ is at most countable), then condition (1) implies the strong measurability of $h$. (See [2, Proposition 6].)

Through the article we fix a triple $(X, \mathcal{N}, \mathcal{I})$ as above and a Banach space $Z$. We assume that condition (ccc) is fulfilled in $(X, \mathcal{M}, \mathcal{I})$.

Let $(Y, \mathcal{N}, \nu)$ be a measure space. We will consider the product $\sigma$-ideal $\widetilde{\mathcal{I}}$ (the family of all subsets of the sets of the form $A \times Y$, where $A \in \mathcal{I}$ ) and the product $\sigma$-field $\mathcal{N} \otimes \mathcal{N}$ (the smallest $\sigma$-field containing the $\sigma$-ideal $\widetilde{\mathcal{I}}$ and the family of all sets of the form $M \times N$, where $M \in \mathcal{M}$ and $N \in \mathcal{N})$.

We assume that we have a net structure $\mathcal{J}$ in $(Y, \mathcal{N}, \nu)$ (see, e.g., [1]). Recall that a net in $Y$ is an at most countable cover of $Y$ consisting of 
pairwise disjoint measurable sets of positive finite measure. The individual sets in the net are called cells. The family $\mathcal{J}=\bigcup_{n=1}^{\infty} \mathcal{J}_{n}$, where each $\mathcal{J}_{n}$ is a net, is called a net structure. Observe that for each $y \in Y$ and $n \in \mathbb{N}$, there is a unique cell from the net $\mathcal{J}_{n}$ which contains $y$. We will denote this cell by $J_{n}(y)$. Several examples of net structures can be found, e.g., in [4] or [5].

A function $g: Y \rightarrow Z$ is called a derivative (with respect to the net structure $\mathcal{J}$ ), if $g$ is Bochner integrable over each $J \in \mathcal{J}$ and for each $y \in Y$,

$$
\lim _{n \rightarrow \infty} \frac{1}{\nu\left(J_{n}(y)\right)} \int_{J_{n}(y)} g=g(y) .
$$

Let $\mathcal{F} \subset Z^{Y}$ be a family of Bochner integrable functions. We say that the functions in $\mathcal{F}$ are equiderivatives at a point $y \in Y$ (with respect to the net structure $\mathcal{J}$ ) [3], if for each $\varepsilon>0$ we can find an $N \in \mathbb{N}$ such that for all $n>N$ and $f \in \mathcal{F}$,

$$
\left\|\frac{1}{\nu\left(J_{n}(y)\right)} \int_{J_{n}(y)} f-f(y)\right\|<\varepsilon .
$$

We will say that a family $\mathcal{A} \subset \mathcal{J}$ has property (V) in a set $J$, if for each $y \in J$ and each $n \in \mathbb{N}$, there is an $m>n$ with $J_{m}(y) \in \mathcal{A}$. We define the following property, pertaining to a net structure $\mathcal{J}$ :

for each $J \in \mathcal{J}$, each family $\mathcal{A} \subset \mathcal{J}$ which possesses property (V)

in $J$, and each $\varepsilon>0$, we can choose pairwise disjoint sets $A_{1}, \ldots, A_{k} \in \mathcal{A}$ such that $\nu\left(J \triangle \bigcup_{i=1}^{k} A_{i}\right)<\varepsilon$.

This property is a generalization of the Vitali property for intervals in $\mathbb{R}^{m}$.

Example 1. Let $\mathcal{J}=\bigcup_{n \in \mathbb{N}} \mathcal{J}_{n}$, where $\mathcal{J}_{n}$ denotes the family of all intervals of the form

$$
\left[\frac{i_{1}-1}{2^{n}}, \frac{i_{1}}{2^{n}}\right) \times \cdots \times\left[\frac{i_{m}-1}{2^{n}}, \frac{i_{m}}{2^{n}}\right),
$$

where $i_{1}, \ldots, i_{m}$ are integers. Then $\mathcal{J}$ possesses property (2).

Theorem 2. Assume that $\mathcal{J}$ possesses property (2). Let $g: X \times Y \rightarrow Z$ be a bounded function, whose all horizontal sections $g^{y}$ are strongly measurable with respect to $(\mathcal{M}, \mathcal{I})$, while all vertical sections $g_{x}$ are equiderivatives at each $y \in Y$. Then $g$ is strongly measurable with respect to $(\mathcal{M} \otimes \mathcal{N}, \widetilde{\mathcal{I}})$.

Proof. Let $M \in \mathbb{R}$ be such that $\|g(x, y)\|<M$ for each $(x, y) \in X \times Y$. Let $J \in \mathcal{J}$. Define a function $h_{J}: X \rightarrow Z$ by $h_{J}(x)=\int_{J} g_{x}$. We will use Remark 3 to show that $h_{J}$ is strongly measurable with respect to $(\mathcal{M}, \mathcal{I})$. 
Fix $\varepsilon>0$ and $A \in \mathcal{M} \backslash \mathcal{I}$. Since the vertical sections of $g$ are equiderivatives, for each $y \in Y$ there is an $N(y) \in \mathbb{N}$ such that for all $n>N(y)$ and $x \in X$,

$$
\left\|\frac{1}{\nu\left(J_{n}(y)\right)} \int_{J_{n}(y)} g_{x}-g_{x}(y)\right\|<\frac{\varepsilon}{16 \nu(J)}
$$

Clearly the family $\mathcal{A}=\left\{J_{n}(y): y \in J, n>N(y)\right\}$ possesses the property (V) in $J$. By assumption (2), we can choose $y_{1}, \ldots, y_{k} \in J$ and $n_{1}, \ldots, n_{k} \in \mathbb{N}$ such that the cells $J_{n_{1}}\left(y_{1}\right), \ldots, J_{n_{k}}\left(y_{k}\right)$ are pairwise disjoint, $n_{i}>N\left(y_{i}\right)$ for each $i$, and

$$
\nu(K \cup L)<\min \left\{\frac{\varepsilon}{4 M}, \nu(J)\right\},
$$

where

$$
K=J \backslash \bigcup_{i=1}^{k} J_{n_{i}}\left(y_{i}\right), \quad L=\bigcup_{i=1}^{k} J_{n_{i}}\left(y_{i}\right) \backslash J .
$$

Recall that all sections $g^{y_{i}}$ are strongly measurable. So, using $k$ times Proposition 1 we can find a set $B \in \mathcal{M} \backslash \mathcal{I}$ with $B \subset A$ such that for all $v, w \in B$ and $i \in\{1, \ldots, k\}$,

$$
\left\|g^{y_{i}}(v)-g^{y_{i}}(w)\right\|<\frac{\varepsilon}{8 \nu(J)} .
$$

Consequently, for all $v, w \in B$,

$$
\begin{aligned}
& \left\|h_{J}(v)-h_{J}(w)\right\|=\left\|\int_{J} g_{v}-\int_{J} g_{w}\right\|=\left\|\int_{J}\left(g_{v}-g_{w}\right)\right\| \\
& =\left\|\sum_{i=1}^{k} \int_{J_{n_{i}}\left(y_{i}\right)}\left(g_{v}-g_{w}\right)+\int_{K}\left(g_{v}-g_{w}\right)-\int_{L}\left(g_{v}-g_{w}\right)\right\| \\
& \leq \sum_{i=1}^{k}\left\|\int_{J_{n_{i}}\left(y_{i}\right)}\left(g_{v}-g_{w}\right)\right\|+\int_{K \cup L}\left\|g_{v}-g_{w}\right\| \\
& \leq \sum_{i=1}^{k}\left\|\int_{J_{n_{i}}\left(y_{i}\right)}\left(g_{v}-g\left(v, y_{i}\right)\right)\right\|+\sum_{i=1}^{k}\left\|\int_{J_{n_{i}}\left(y_{i}\right)}\left(g\left(v, y_{i}\right)-g\left(w, y_{i}\right)\right)\right\| \\
& \quad+\sum_{i=1}^{k}\left\|\int_{J_{n_{i}\left(y_{i}\right)}}\left(g\left(w, y_{i}\right)-g_{w}\right)\right\|+2 M \frac{\varepsilon}{4 M}
\end{aligned}
$$




$$
\begin{aligned}
\leq & \sum_{i=1}^{k} \nu\left(J_{n_{i}}\left(y_{i}\right)\right)\left\|\frac{1}{\nu\left(J_{n_{i}}\left(y_{i}\right)\right)} \int_{J_{n_{i}}\left(y_{i}\right)} g_{v}-g_{v}\left(y_{i}\right)\right\| \\
& +\sum_{i=1}^{k} \int_{J_{n_{i}}\left(y_{i}\right)}\left\|g\left(v, y_{i}\right)-g\left(w, y_{i}\right)\right\| \\
& \quad+\sum_{i=1}^{k} \nu\left(J_{n_{i}}\left(y_{i}\right)\right)\left\|\frac{1}{\nu\left(J_{n_{i}}\left(y_{i}\right)\right)} \int_{J_{n_{i}}\left(y_{i}\right)} g_{w}-g_{w}\left(y_{i}\right)\right\|+\frac{\varepsilon}{2} \\
\leq & \sum_{i=1}^{k} \nu\left(J_{n_{i}}\left(y_{i}\right)\right)\left(\frac{\varepsilon}{16 \nu(J)}+\frac{\varepsilon}{8 \nu(J)}+\frac{\varepsilon}{16 \nu(J)}\right)+\frac{\varepsilon}{2} \\
= & \nu\left(\bigcup_{i=1}^{k} J_{n_{i}}\left(y_{i}\right)\right) \frac{\varepsilon}{4 \nu(J)}+\frac{\varepsilon}{2} \\
\leq & \nu(J \cup L) \frac{\varepsilon}{4 \nu(J)}+\frac{\varepsilon}{2} \leq \frac{\varepsilon}{4}+\nu(L) \frac{\varepsilon}{4 \nu(J)}+\frac{\varepsilon}{2}<\varepsilon .
\end{aligned}
$$

We have proved that $h_{J}$ fulfills condition (1). By Remark $3, h_{J}$ is strongly measurable with respect to $(\mathcal{M}, \mathcal{I})$. Now repeating the last part of the proof of $[2$, Theorem 7$]$ we can show that $g$ is strongly measurable as well.

Corollary 3. Assume that J possesses property (2). Let $f: X \times Y \rightarrow Z$ be a bounded function, whose all horizontal sections $f^{y}$ are strongly measurable with respect to $(\mathcal{M}, \mathcal{I})$. Assume moreover that there is a set $S \in \mathcal{I}$ such that the vertical sections $f_{x}, x \in X \backslash S$, are equiderivatives at each $y \in Y$. Then $f$ is strongly measurable with respect to $(\mathcal{M} \otimes \mathcal{N}, \widetilde{\mathcal{I}})$.

Proof. Define

$$
g(x, y)= \begin{cases}f(x, y) & \text { if } x \in X \backslash S \\ 0 & \text { if } x \in S\end{cases}
$$

One can easily see that $g$ fulfills the assumptions of Theorem 2 , so $g$ is strongly measurable with respect to $(\mathcal{M} \otimes \mathcal{N}, \widetilde{\mathcal{I}})$. Since the equality $f=g$ holds $\widetilde{\mathcal{I}}$-almost everywhere, $f$ is strongly measurable as well.

Now assume that $\mathcal{I}=\{A \in \mathcal{M}: \mu(A)=0\}$, where $\mu: \mathcal{M} \rightarrow[0, \infty]$ is a nontrivial measure, and that $\mathcal{K}=\bigcup_{m=1}^{\infty} \mathcal{K}_{m}$ is a net structure in $X$. For each $x \in X$ and $m \in \mathbb{N}$ we denote by $K_{m}(x)$ the unique cell from the net $\mathcal{K}_{m}$ which contains $x$.

Theorem 4. Assume that $\mathcal{J}$ possesses property (2). Let $f: X \times Y \rightarrow Z$ be a bounded function, whose all horizontal sections $f^{y}$ are derivatives with 
respect to $\mathcal{K}$, while all vertical sections $f_{x}$ are equiderivatives at each $y \in Y$. Then $f$ is a derivative in the strong sense, i.e., for each $(x, y) \in X \times Y$,

$$
\lim _{\substack{m \rightarrow \infty \\ n \rightarrow \infty}} \frac{1}{\mu\left(K_{m}(x)\right) \nu\left(J_{n}(y)\right)} \int_{K_{m}(x) \times J_{n}(y)} f(u, v) d(\mu \times \nu)(u, v)=f(x, y) .
$$

Proof. Fix $(x, y) \in X \times Y$ and $\varepsilon>0$. Since all vertical sections $f_{u}$ are equiderivatives at $y$, there is an $N \in \mathbb{N}$ such that for all $n>N$ and $u \in X$,

$$
\left\|\frac{1}{\nu\left(J_{n}(y)\right)} \int_{J_{n}(y)} f_{u}(v) d \nu(v)-f_{u}(y)\right\|<\frac{\varepsilon}{2} .
$$

Since $f^{y}$ is a derivative at $x$, there is an $M \in \mathbb{N}$ such that for each $m>M$,

$$
\left\|\frac{1}{\mu\left(K_{m}(x)\right)} \int_{K_{m}(x)} f^{y}(u) d \mu(u)-f^{y}(x)\right\|<\frac{\varepsilon}{2} .
$$

By Theorem 2, function $f$ is strongly measurable. So, it is Bochner integrable on $K_{m}(x) \times J_{n}(y)$. (See, e.g., [6, p. 133].) Using the Fubini Theorem, for all $n>N$ and $m>M$ we obtain

$$
\begin{aligned}
& \left\|\frac{1}{\mu\left(K_{m}(x)\right) \nu\left(J_{n}(y)\right)} \int_{K_{m}(x) \times J_{n}(y)} f(u, v) d(\mu \times \nu)(u, v)-f(x, y)\right\| \\
& =\left\|\frac{1}{\mu\left(K_{m}(x)\right) \nu\left(J_{n}(y)\right)} \int_{K_{m}(x)}\left(\int_{J_{n}(y)} f_{u}(v) d \nu(v)\right) d \mu(u)-f(x, y)\right\| \\
& \leq\left\|\frac{1}{\mu\left(K_{m}(x)\right)} \int_{K_{m}(x)}\left(\frac{1}{\nu\left(J_{n}(y)\right)} \int_{J_{n}(y)} f_{u}(v) d \nu(v)-f_{u}(y)\right) d \mu(u)\right\| \\
& \quad+\left\|\frac{1}{\mu\left(K_{m}(x)\right)} \int_{K_{m}(x)} f_{u}(y) d \mu(u)-f(x, y)\right\| \\
& \leq \frac{1}{\mu\left(K_{m}(x)\right)} \int_{K_{m}(x)}\left\|\frac{1}{\nu\left(J_{n}(y)\right)} \int_{J_{n}(y)} f_{u}(v) d \nu(v)-f_{u}(y)\right\| d \mu(u) \\
& \quad+\left\|\frac{1}{\mu\left(K_{m}(x)\right)} \int_{K_{m}(x)} f^{y}(u) d \mu(u)-f^{y}(x)\right\| \\
& \leq \frac{1}{\mu\left(K_{m}(x)\right)} \frac{\varepsilon}{2} \mu\left(K_{m}(x)\right)+\frac{\varepsilon}{2}=\varepsilon .
\end{aligned}
$$

Remark 4. If $\mathcal{J}=\mathcal{K}$ is the net structure from Example 1 (for $m=1$ ), $\mathcal{M}$ is the $\sigma$-field of Lebesgue measurable sets, $\mathcal{I}$ is the $\sigma$-ideal of null sets, and $\mu=\nu$ is the Lebesgue measure, then Corollary 3 and Theorem 4 are precisely the main results of [3]. 


\section{References}

[1] Bruckner, A. M., Differentiation of integrals, p. II, Amer. Math. Monthly 78(9) (1971).

[2] Chmielewska, K., On the strong product measurability, Real Anal. Exchange 26(1) (2000-01), 437-443.

[3] Grande, Z., On equi-derivatives, Real Anal. Exchange 21(2) (1995-96), 637-647.

[4] Mišík, L., Über den Mittelwertsatz für additive Zellenfunktionen, Mat.-Fyz. Casopis. Sloven. Acad. Vied 15(4) (1963), 260-274.

[5] Pauc, C., Dérivées et Intégrales: Fonctions de cellule, Conférence faite au Centre Mathématique International de Varenna, 15-25 Aug. 1954, pub. Mathematical Institute, Rome.

[6] Yosida, K., Functional Analysis, Springer-Verlag, Berlin-Heidelberg-New York, 1980.

Katarzyna Chmielewska

Institute of MATHEMATics

Bydgoszcz ACAdemy

PL. WEYSSENHOFFA 11

85-072 BYDGOSZCZ

POLAND

EMAIL: KASIACH@AB-BYD.EDU.PL 